\title{
A Comparison of Stent Implant versus Medical Treatment for Severe Symptomatic Intracranial Stenosis: A Controlled Clinical Trial
}

\author{
Rezao Mohammadian ${ }^{\mathrm{a}}$ Ali Pashapour ${ }^{\mathrm{a}}$ Ehsan Sharifipour ${ }^{\mathrm{a}}$ \\ Reza Mansourizadeh ${ }^{\mathrm{b}}$ Farideh Mohammadian ${ }^{\mathrm{a}}$ \\ Ali Akbar Taher Aghdam. ${ }^{a}$ Mohammad Mousavi ${ }^{a}$ \\ Farhad Dadras ${ }^{\mathrm{C}}$ \\ a Neuroscience Research Center, Tabriz University of Medical Sciences, \\ ${ }^{\mathrm{b}}$ Neurology Department, Alinasab Hospital, and ${ }^{\mathrm{C}}$ Neurology Department, \\ Mahalati Hospital, Tabriz, Iran
}

\section{Key Words}

Ischemic stroke - Intracranial stenosis - Balloon-mounted coronary bare metal stents • Self-expandable stents $\cdot$ Functional outcome $\cdot$ Primary adverse events $\cdot$ Secondary adverse events

\begin{abstract}
Background: Atherosclerotic stenosis of the major intracranial arteries is the most common cause of ischemic stroke. There are limited treatments for severe intracranial stenosis, and stent placement versus medical treatment remains controversial. The aim of this study was to compare functional outcomes of these two modalities in patients with severe symptomatic intracranial stenosis. Methods: At a single center, between 2008 and 2011, patients with angiographically demonstrated severe (70-90\%) symptomatic intracranial atherosclerosis were divided into two groups: group A, which received only medical treatment, and group B, which underwent endovascular stent implant treatment. The severity and location of the stenosis was determined by digital subtraction angiography and the Warfarin-Aspirin Symptomatic Intracranial Disease (WASID) trial criteria in all patients. The exclusion criteria were: specific causes other than atherosclerosis, such as artery dissection, fibromuscular dysplasia, vasculitis, radiation and intracranial hemorrhage, focal neurological deficit that did not correlate to internal carotid artery or
\end{abstract}




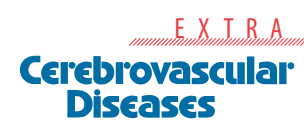

Cerebrovasc Dis Extra 2012;2:108-120

Mohammadian et al.: A Comparison of Stent Implant versus Medical Treatment for Severe Symptomatic Intracranial Stenosis: A Controlled Clinical Trial

middle cerebral artery stenosis. All procedures were done under light anesthesia. Technical success was defined as the reduction of stenosis to $<30 \%$ with complete enveloping of the lesion after the procedure. Early and late adverse events and functional outcomes were compared between the groups using the modified Rankin Scale (mRS). Results: Overall, 63 patients (29 in group A and 34 in group B) were evaluated and followed for a mean period of 15.22 months (range 6-25). The technical success rate was $97 \%$ in a total of 34 stents in 34 patients. There was no difference between the early (within 30 days) adverse event rates of the two groups. The median follow-up duration for the stent implant patients was 15 months (range 6-25), and for the medically treated cohort it was 14 months (range $8-25$ ). The re-stenosis rate was $5.8 \%$ and the total number of late ( $>30$ days) adverse events, including stroke, myocardial infarction and death, was $1(2.9 \%)$ and $6(20.7 \%)$ in the stent implant and medical groups, respectively $(p=$ 0.042). The stent implant group had significantly better favorable functional outcomes according to the $\mathrm{mRS}$ than the medical group (93.9 vs. $63.0 \%$ ). The cumulative secondary adverse event-free survival was significantly lower in the stent implant group. Conclusion: Stent implants can be considered more durable and safe for patients with symptomatic severe stenosis of the internal carotid artery or middle cerebral artery, despite optimal medical therapy. Randomized, multicenter trials are required to confirm these results.

Copyright $\odot 2012$ S. Karger AG, Basel

\section{Introduction}

Stroke is the third leading cause of death and adult disability in North America, Europe, and Asia $[1,2]$. Around the world, the most common cause of ischemic stroke is probably atherosclerotic stenosis of the major intracranial arteries [3, 4]. Patients with a similar degree of arterial stenosis may have a different prognosis depending on the ensuing cerebral flow status [5]. There are limited options for treatment of intracranial stenosis. Medical therapy with antiplatelet and antithrombotic agents has been widely used, but there is a high rate of failure, and such problems have spurred interest in endovascular treatment approaches [6]. However, a recent historical cohort study on severe intracranial stenosis of 70-99\% demonstrated that aggressive medical management was superior to endovascular stent implantation $[7,8]$. Considering these uncertainties regarding the safety and efficacy of medical therapy alone, as compared with intracranial stent implantation, we designed this study to compare the outcomes of these two therapeutic approaches in patients with severe (70-90\%) symptomatic intracranial stenosis in the territory of the middle cerebral artery (MCA) or internal carotid artery (ICA) presenting with transient ischemic attack (TIA) or ischemic stroke.

\section{Materials and Methods}

\section{Subjects and Procedures}

Sixty-three patients with symptomatic intracranial arterial stenosis, presenting with ischemic stroke or TIA, were referred to our institute and evaluated prospectively in a nonrandomized study from September 2008 to December 2011. The diagnosis of ischemic stroke was based on a focal neurologic deficit with corresponding ischemic lesions demonstrated with spiral brain computed tomography (CT) and brain magnetic resonance imaging (MRI) after admission. Those that had severe (70-99\%) stenosis of the intracranial ICA or MCA branches by magnetic resonance angiography (MRA) within the previous 21 days were included in our study (using convenient sampling). The severity and location of the stenosis 
was determined by digital subtraction angiography and the Warfarin-Aspirin Symptomatic Intracranial Disease (WASID) trial criteria [9] in all patients.

The intensity of the neurological deficit at admission was assessed by neurologists using the National Institutes of Health Stroke Scale (NIHSS). We non-randomly categorized patients into two groups with an allocation sequence: the medical group, which received only medical treatment, and the stent implant group, which underwent endovascular balloon-mounted coronary bare metal stent (BMS) placement in a stenosis at the intracranial ICA and self-expandable stent (SES) placement in a MCA stenosis. The treatment decision was thoroughly evaluated by a team of neurologists and a neuroradiologist. The exclusion criteria were: specific causes other than atherosclerosis, such as artery dissection, fibromuscular dysplasia, vasculitis, radiation and intracranial hemorrhage, focal neurological deficit that did not correlate to ICA or MCA occlusion, a type C of Mori et al. [10] in MCA stenosis, and patients who underwent only endovascular angioplasty. For a matched comparison and to avoid selection bias, the vascular risk factors, anatomic location of the stenosis, stenosis degree, and baseline modified Rankin Scale (mRS) score and NIHSS score were all matched between the two groups (by frequency matching), as revealed in table 1 .

A standard protocol was used for evaluation of all patients. Demographic features and vascular risk factors were recorded by an expert neurologist, including hypertension, diabetes mellitus, ischemic heart disease, dyslipidemia (defined as receiving lipid-lowering agents or having an overnight fasting cholesterol level $>200 \mathrm{mg} / \mathrm{dl}$ and low-density lipoprotein $>100$ $\mathrm{mg} / \mathrm{dl}$ ), cigarette smoking, and previous TIA or stroke (table 1).

All paraclinical data of patients were evaluated as well, including red and white blood cell and platelet counts, blood glucose and glycosylate hemoglobin (HbAlc) levels, lipid profiles, serum electrolyte levels, liver transaminase levels, blood urea and creatinine levels, measurement of prothrombin and activated partial thromboplastin times, and evaluation with electrocardiography (data not shown). All patients received standard care ordered by the stroke care unit (antiplatelet, statin, and daily physiotherapy) and were usually discharged during 1 week after admission by a neurologist. After this time, the patients were recalled and a skilled neuroradiologist informed the patients about the benefits and potential risks of the endovascular stent implant therapy and offered them this treatment as an alternative. Patients who gave informed consent were transferred to the angiography room. These patients were required to have no contraindications for endovascular procedures (renal failure, coagulopathy, and contrast allergy). The rationale for the medical treatment of patients was that they refused the stent implant because of the procedural risk and/or the expense, although they were stent qualified. Also, a few patients desired a stent but had not been offered a stent implant due to technical difficulties.

Endovascular angioplasty and stent implantations were performed by the same interventional neuroradiologist that implemented angiography. All procedures were done under light anesthesia. All collateral flow to the infarcted cerebral hemisphere was systematically assessed. The artery that was clinically assumed to be involved was investigated last and, after this diagnostic angiography, endovascular therapy was performed directly.

A 7F guide catheter was placed into the proximal cervical ICA. The shortest coronary BMS that covered the full length of the stenotic segment was chosen, and the same technique for stenting of proximal ICA stenosis was deployed. However, for MCA stenosis, we used a SES. All patients received clopidogrel $75 \mathrm{mg} /$ day and ASA $100 \mathrm{mg} /$ day at least 3 days previous to the procedure and full heparinization during the procedure. ICA stenoses were distinguished with respect to the affected segment and included stenosis of the intracranial segment of the ICA from the initiation of the petrous segments up to the communicating segments, and MCA stenoses were classified as stenosis of this artery from the origin to the 

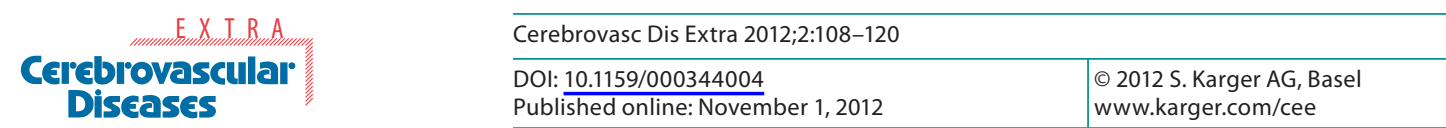

Mohammadian et al.: A Comparison of Stent Implant versus Medical Treatment for Severe Symptomatic Intracranial Stenosis: A Controlled Clinical Trial

Table 1. Patient characteristics according to treatment protocol

\begin{tabular}{|c|c|c|c|}
\hline Characteristics & $\begin{array}{l}\text { Stent-implanted } \\
\text { group }\end{array}$ & $\begin{array}{l}\text { Medically treated } \\
\text { group }\end{array}$ & $\mathrm{p}$ value \\
\hline Patients & $34(54.0)$ & $29(46.0)$ & \\
\hline Gender & & & 0.793 \\
\hline Male & $20(58.8)$ & $18(62.1)$ & \\
\hline Female & $14(41.2)$ & $11(37.9)$ & \\
\hline Age, years & $67.88 \pm 8.42$ & $70.34 \pm 6.99$ & 0.484 \\
\hline \multicolumn{4}{|l|}{ Vascular risk factors } \\
\hline Diabetes & $24(70.6)$ & $17(58.6)$ & 0.321 \\
\hline Hypertension & $24(70.6)$ & $20(69.0)$ & 0.889 \\
\hline Dyslipidemia & $4(11.7)$ & $6(20.6)$ & 0.677 \\
\hline Cigarette smoking & $12(35.2)$ & $15(51.7)$ & 0.198 \\
\hline Ischemic heart disease & $11(32.4)$ & $14(48.3)$ & 0.247 \\
\hline Previous TIA or stroke & $4(11.7)$ & $3(10.3)$ & 0.289 \\
\hline Presenting symptoms & & & 0.987 \\
\hline Ischemic stroke & $20(58.8)$ & $17(58.6)$ & \\
\hline TIA & $14(41.2)$ & $12(41.4)$ & \\
\hline Lesion locations & & & 0.233 \\
\hline ICA & $18(52.9)$ & $11(37.9)$ & \\
\hline MCA & $16(47.1)$ & $18(62.1)$ & \\
\hline Location of MCA occlusion & & & 0.356 \\
\hline M1 segment & $9(26.5)$ & $10(34.5)$ & \\
\hline M2 segment & $7(20.6)$ & $8(27.6)$ & \\
\hline \multicolumn{4}{|l|}{ Location of ICA occlusion } \\
\hline Cavernous & $8(23.5)$ & $8(27.6)$ & \\
\hline Petrous & $7(20.6)$ & $2(6.9)$ & \\
\hline Petrocavernous & $3(8.8)$ & $1(3.4)$ & \\
\hline Length of stenosis ( $\geq 7 \mathrm{vs} .<7 \mathrm{~mm}$ ) & $18(52.9)$ & $12(41.3)$ & 0.147 \\
\hline Functional status (initial mRS score) & & & 0.867 \\
\hline 2 & $4(11.8)$ & $2(6.9)$ & \\
\hline 3 & $14(42.2)$ & $12(14.4)$ & \\
\hline 4 & $6(17.6)$ & $7(24.1)$ & \\
\hline 5 & $10(29.4)$ & $8(27.6)$ & \\
\hline Initial NIHSS & $7.82 \pm 3.05$ & $7.59 \pm 3.14$ & 0.782 \\
\hline Stenosis degree at presentation, \% & $81.65 \pm 1.26^{*}$ & $77.09 \pm 1.45^{*}$ & 0.062 \\
\hline Stenosis degree distribution & & & 0.055 \\
\hline $70-80 \%$ & $20(58.8)$ & $24(82.8)$ & \\
\hline $81-90 \%$ & $14(41.2)$ & $5(17.2)$ & \\
\hline Follow-up duration, months & $15.18 \pm 4.80$ & $15.28 \pm 4.76$ & 0.802 \\
\hline
\end{tabular}

Data are presented as means \pm SD or numbers with percentages in parentheses. $M 1=$ First segment of the MCA artery; M2 = second segment of the MCA artery.

${ }^{*}$ Data are presented as means \pm standard error.

origin of the cortical branches of the horizontal (M1) and Sylvain (M2) segments, based on Ziyal et al. [11] and Mori et al. [10] nomenclature, respectively. To prevent hyperperfusion, patients were transferred to the intensive care unit for strict control of blood pressure and their general condition. To exclude any asymptomatic complications, brain MRI, including diffusion-weighted imaging and apparent diffusion coefficient mapping, was performed within $72 \mathrm{~h}$ following intervention. 


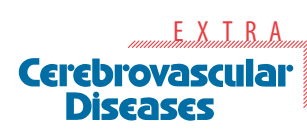

Cerebrovasc Dis Extra 2012;2:108-120

DOI: $10.1159 / 000344004$

Published online: November 1, 2012

Mohammadian et al.: A Comparison of Stent Implant versus Medical Treatment for Severe Symptomatic Intracranial Stenosis: A Controlled Clinical Trial

Following the initial diagnosis, the medical group received double the antiplatelet regimen (aspirin $80 \mathrm{mg} /$ daily, clopidogrel $75 \mathrm{mg} /$ daily) for lifetime and atorvastatin $(20 \mathrm{mg} /$ daily) for at least 12 months. After the procedure, patients received the same treatment. All patients in each group underwent daily physiotherapy (if needed) by standard protocols in the same center. None of them switched to the other group.

Both groups were managed similarly and followed up clinically at 1 month and every 6 months after study enrolment until the end of the follow-up time, which was when the last included patient had been followed for 6 months. According to this definition, 5 patients in the medical group [ 3 had ICA severe stenosis ( 2 in cavernous and 1 in petrous segments) and 2 had MCA severe stenosis ( 1 in M1 and 1 in M2 segments), all of them by digital subtraction angiography], who did not agree with continuing follow-up, exited the study before the planned 6 months for follow-up MRI or transcranial Doppler (TCD), and their data were not evaluated. The follow-up exams were performed by independent neurologists (who were not involved in the interventional procedures) via outpatient visits and vascular risk factor management. During these visits, if new neurological signs occurred, which were suspected to be a stroke, the patient was examined by the study neurologist for future study by MRI or brain CT. Within the follow-up period, high blood pressure (systolic blood pressure was preferred to be $<140 \mathrm{~mm} \mathrm{Hg}$, or $<130 \mathrm{~mm} \mathrm{Hg}$ if the patient was diabetic) and high blood sugar were treated aggressively and patients who smoked were asked to cease cigarette smoking along with participating in lifestyle modification programs including weight reduction, exercise, and maintaining a nutritional diet.

Technical success was defined as the reduction of stenosis to $<30 \%$ with complete enveloping of the lesion after the procedure [12]. The stent implant group had scheduled follow-up brain MRI, MRA or TCD at 6 and 12 months after the procedure for examination of potential re-stenosis. In-stent re-stenosis was described as recurrent artery stenosis to the degree of $>30 \%$ luminal narrowing after excluding post-stent implant residual stenosis. The re-stenosis degree and location were determined based on the suggested method of Samuels et al. [13] and increased flow velocity on follow-up TCD below or above the stent-implemented vessels. The occurrence of clinical adverse events (AEs) for all patients was evaluated 30 days after enrolment or after stent implantation, and then every 6 months until follow-up ended. The AEs were classified as occurring within 30 days and follow-up events. The within-30days AEs were classified as happening during the 30 days after enrolment, including ischemic stroke in the territory or in another zone of the qualifying artery, symptomatic intracranial hemorrhage, and TIA. Follow-up AEs (FAEs) were defined as arising 30 days after enrolment during the follow-up period. The FAEs consisted of ischemic stroke (stroke in the territory of the qualifying artery), TIA, myocardial infarction, and mortality related to these events. In addition, the patients were informed of suggestive symptoms of AEs, and they were instructed to visit their physician as soon as possible if the 6-month visit had not occurred. The causes of death were obtained from relatives of the deceased patients. The mRS scores were calculated at the end of the follow-up period. Scores of $0-2$ were classified as a favorable functional status and scores of 3-5 were categorized as an unfavorable functional status. Mortality was indicated by a score of 6 .

\section{Statistical Method}

The study was designed to have $>80 \%$ power to detect a $35 \%$ difference in the base of $5 \%$ change in the mRS score. Numerical variables are presented as mean $\pm \mathrm{SD}$ and categorical variables were summarized by absolute frequencies and percentages. Continuous variables were compared using the Student's t test or a non-parametric Mann-Whitney U test whenever the data did not appear to have a normal distribution. Categorical variables were compared using a $\chi^{2}$ or Fisher's exact test. The observation time was from the study enrolment 
to within-30-days AEs, FAE occurrence, loss to follow-up, or the patient being event-free until 25 months. Univariate analyses for comparing within-30-days AEs and FAEs across the two groups of patients were then established and the associations are presented as odds ratios (ORs) with $95 \%$ confidence intervals (CIs). Time to events was analyzed by Kaplan-Meier and log-rank tests to compare the cumulative event rates and functional outcomes of the two groups. The proportional hazard assumption was checked to apply to the methods. For statistical analyses, the statistical software SPSS version 13.0 for Windows (SPSS Inc., Chicago, Ill., USA) was used. All $\mathrm{p}$ values were two-tailed, with statistical significance defined by $\mathrm{p} \leq$ 0.05 .

\section{Results}

Overall, 63 patients were evaluated. For all patients, the mean age was $69.02 \pm 7.72$ years (range 44-82) and male gender was predominant (60.3\%). Stents were implanted in 34 (54\%) patients. There were no significant differences between the stent implant group and 29 matched patients in the medically managed group in terms of basic characteristics, follow-up time, baseline functional status, stenosis location, and degree of stenosis (all p values were $>0.05$; table 1). Overall, hypertension was found in $69.8 \%$ of the patients and was the most common vascular risk factor. A similar incidence was found for the presenting symptoms in the two groups including TIA and stroke (41.2 and 58.7\%, respectively). ICA was involved in $46.0 \%$ of the patients, predominantly at the cavernous segment $(25.3 \%)$, and MCA was involved in $53.9 \%$ of the patients, frequently at the M1 segment (30.1\%). The mean degree of stenosis was approximately $79 \%$ at presentation in all patients, which was slightly higher in the stent implant group. Eighteen (52.9\%) BMSs were implanted for ICA stenosis, while 16 (47.1\%) SESs were implanted for MCA stenosis. There were no statistically significant differences in within-30-days AEs, FAEs, and re-stenosis rates between the two groups (data not shown). During the procedure, 6 patients had hypotension and bradycardia, which were treated with inotropes. Hypertension occurred in 2 patients who were treated with antihypertensive agents. All of these incidents were reversible, with complete recovery within $24 \mathrm{~h}$ of the procedure. The technical success rate was $97 \%$ in a total of 34 stents in 34 patients. The overall mean follow-up time was $15.22 \pm 4.74$ months. The median follow-up duration for the stent implant patients was 15 months (range 6-25) and for the medically treated cohort was 14 months (range 8-25). The in-stent re-stenosis rate in our study was 5.8\%, which happened in 2 patients. In 1 patient, re-stenosis occurred 12 months after the procedure in the M1 segment stent and in the other patient at 15 months in the cavernous segment. Both patients were asymptomatic. The estimation of stenosis advancement was troublesome for the medically managed group because performing TCD and MRA were not desired during the follow-up period. The overall rate of within-30-days AEs in our study was $3.1 \%$ and there was no statistically significant difference in within-30-days AEs between the two groups ( $\mathrm{p}=$ 0.909 ; table 2). The total incidence rate of FAEs in our survey was $11.1 \%$, which was significantly higher $(20.7 \%)$ in the medically managed patients during follow-up ( $p=0.042$; table 2). Most importantly, the stent implant group had significantly more favorable functional outcomes (mRS 2) than the medically managed group. Both groups began with a semiequivalent distribution of baseline mRS (table 1) but terminated with a significant difference in functional outcomes and occurrences of FAEs, as illustrated by the Kaplan-Meier curves (fig. 1 and 2, respectively). The medically managed group had a progressively significant increase in unfavorable outcomes during the time after enrolment ( 93.9 vs. $63.0 \%, p=0.045$; fig. 1) and a significantly lower cumulative 15 -month event-free survival rate (79.3 vs. $97.0 \%$, $\mathrm{p}=0.0243$; fig. 2). 


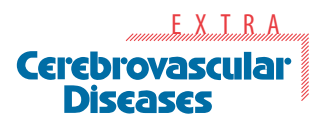

Table 2. Comparison of unfavorable functional status, AEs, and mortality rate between stent-implanted and medically managed group

\begin{tabular}{llll}
\hline Variables & $\begin{array}{l}\text { Stent-implanted } \\
\text { group }(\mathrm{n}=34)\end{array}$ & $\begin{array}{l}\text { Medically treated } \\
\text { group }(\mathrm{n}=29)\end{array}$ & p value \\
\hline Favorable functional status & $31(93.9)$ & $17(63.0)$ & 0.004 \\
mRS 0 & $12(35.3)$ & $3(10.3)$ & \\
mRS 1 & $11(32.4)$ & $8(27.6)$ & \\
mRS 2 & $8(23.5)$ & $6(20.7)$ & 0.909 \\
Within-30-days AEs & $1(2.9)$ & $1(3.4)$ & \\
Ischemic stroke* & $1(2.9)$ & 0 & 0.042 \\
TIA & 0 & $1(3.4)$ & 0.040 \\
FAEs & $1(2.9)$ & $6(20.7)$ & 0.590 \\
Ischemic stroke* & 0 & $4(13.8)^{\mathrm{b}}$ & 0.590 \\
Myocardial infarction & $1(2.9)^{\mathrm{a}}$ & $2(6.9)^{\mathrm{c}}$ & \\
Mortality & $1(2.9)$ & $2(6.9)$ & \\
\hline
\end{tabular}

Data are presented as numbers with percentages in parentheses.

* Stroke in the territory of the qualifying artery.

${ }^{a}$ One 75-year-old male with MCA involvement presenting with ischemic stroke (mRS 2) died 11 months after the procedure because of fatal myocardial infarction.

${ }^{\mathrm{b}}$ Including a 71-year-old male with ICA involvement presenting with TIA (mRS 5) who died 10 months after the study with massive ischemic stroke in the territory of his qualified artery.

${ }^{\mathrm{c}}$ Including a 74-year-old male with MCA involvement presenting with ischemic stroke (mRS 5) who died 12 months after the study because of fatal myocardial infarction.

\section{Discussion}

Atherosclerotic stenosis of the major intracranial arteries is probably the most common cause of stroke [3]. Some ischemic stroke mechanisms that are secondary to intracranial atherosclerosis include intraplaque hemorrhage and the occlusive effect, thromboembolic events distal to the site of the stenosis, direct small penetrating artery occlusion at the site of the plaque and hypoperfusion [14]. Regarding the therapeutic modalities, there are ongoing discussions on the superiority of endovascular stent placement or medical management for intracranial stenosis treatment [15-18]. Despite antiplatelet medications, the annual risk of recurrent stroke is as high as $12 \%$ in patients with symptomatic intracranial atherosclerosis, and stenting and angioplasty have been performed in selected patients with severe stenosis refractory to medical treatment [19]. In this study, we compared within-30-days AEs and FAEs, unfavorable functional status ( $\mathrm{mRS}>2$ ), and event-free survival between the endovascular stent implant and medically managed groups that had angiographically documented severe intracranial stenosis (70-90\%) in the ICA or MCA, with clinical follow-up.

This survey compared within-30-days AEs, FAEs, and unfavorable functional outcomes of 34 ICA or MCA stent-implanted cases with 29 matched medically treated patients. While there were no differences in the total within-30-days AE rate between the two groups (2.9 vs. $3.4 \%, \mathrm{p}=0.909)$, the FAE rate was lower in the stent implant group (2.9\%) than in the medically managed group $(20.7 \%, \mathrm{p}=0.042)$. The 1 -year stroke rate in the medically managed group was $13.8 \%$, which was significantly higher in comparison with the other group during follow-up ( $p=0.040$; table 2$)$. The ischemic strokes in the medically treated group were mostly large cerebral infarctions within the territory of the stenotic artery during the 7-10 months after enrolment (fig. 2). 


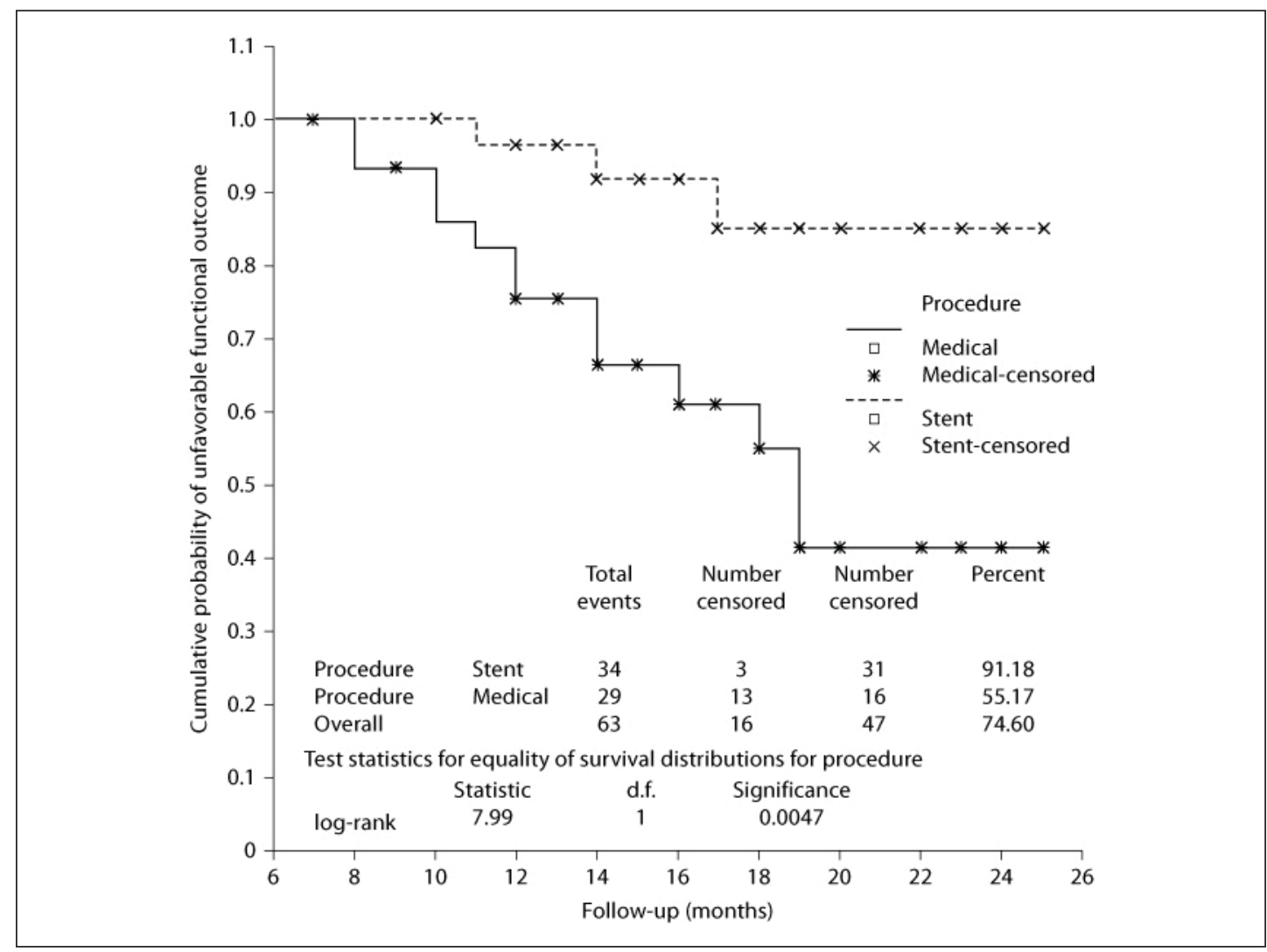

\begin{tabular}{|llllllll|}
\hline $\begin{array}{l}\text { Time } \\
\text { interval } \\
\text { months }\end{array}$ & & $\begin{array}{l}\text { Patients } \\
\text { entering this } \\
\text { interval, } \mathrm{n}\end{array}$ & $\begin{array}{l}\text { Patients with- } \\
\text { drawn during } \\
\text { interval, } \mathrm{n}\end{array}$ & $\begin{array}{l}\text { Patients } \\
\text { exposed } \\
\text { to risk, } \mathrm{n}\end{array}$ & $\begin{array}{l}\text { Unfavorable } \\
\text { functional } \\
\text { outcome, } \mathrm{n}\end{array}$ & $\begin{array}{l}\text { Proportion of } \\
\text { favorable func- } \\
\text { tional outcome }\end{array}$ & $\begin{array}{l}\text { Cumulative probability of } \\
\text { favorable functional out- } \\
\text { come at end of interval, } \%\end{array}$ \\
\hline \multirow{2}{*}{$0-4$} & Medical: & 29 & 0 & 29 & 0 & 1.00 & 100 \\
& Stent: & 34 & 0 & 34 & 0 & 1.00 & 100 \\
\hline \multirow{2}{*}{-8 } & Medical: & 29 & 0 & 29 & 0 & 1.00 & 100 \\
& Stent: & 34 & 3 & 32 & 0 & 1.00 & 100 \\
\hline \multirow{2}{*}{$8-12$} & Medical: & 29 & 1 & 28 & 5 & 0.824 & 82.4 \\
& Stent: & 31 & 2 & 30 & 1 & 0.966 & 96.6 \\
\hline $12-16$ & Medical: & 23 & 6 & 20 & 4 & 0.800 & 65.9 \\
& Stent: & 28 & 12 & 22 & 1 & 0.954 & 92.2 \\
\hline $16-20$ & Medical: & 13 & 4 & 11 & 4 & 0.636 & 41.9 \\
& Stent: & 15 & 8 & 11 & 1 & 0.909 & 83.8 \\
\hline $20-24$ & Medical: & 5 & 3 & 3 & 0 & 1.00 & 41.9 \\
& Stent: & 6 & 4 & 4 & 0 & 1.00 & 83.8 \\
\hline $24-28$ & Medical: & 2 & 2 & 1 & 0 & 1.00 & 41.9 \\
& Stent: & 2 & 2 & 1 & 0 & 1.00 & 83.8 \\
\hline
\end{tabular}

Fig. 1. Kaplan-Meier curve and table of cumulative probability of favorable functional outcome defined as $\mathrm{mRS}$ scores $0-2$. Note that the medically managed group has a markedly worse functional outcome (mRS score 3-5) during follow-up ( $\mathrm{p}=0.0047)$.

The reported rates of periprocedure complications (within 30 days of the procedure) in endovascular stent implant patients have varied from 4.4 to $50 \%$ [20, 21]. In one study, medical care plus angioplasty with or without stent placement was compared with medical care alone. The overall 30-day rate of stroke and mortality was 7.9 and 3.4\%, respectively [22]. In our cases, the periprocedure mortality and stroke rates were 0 and $2.9 \%$ (table 2), which was 

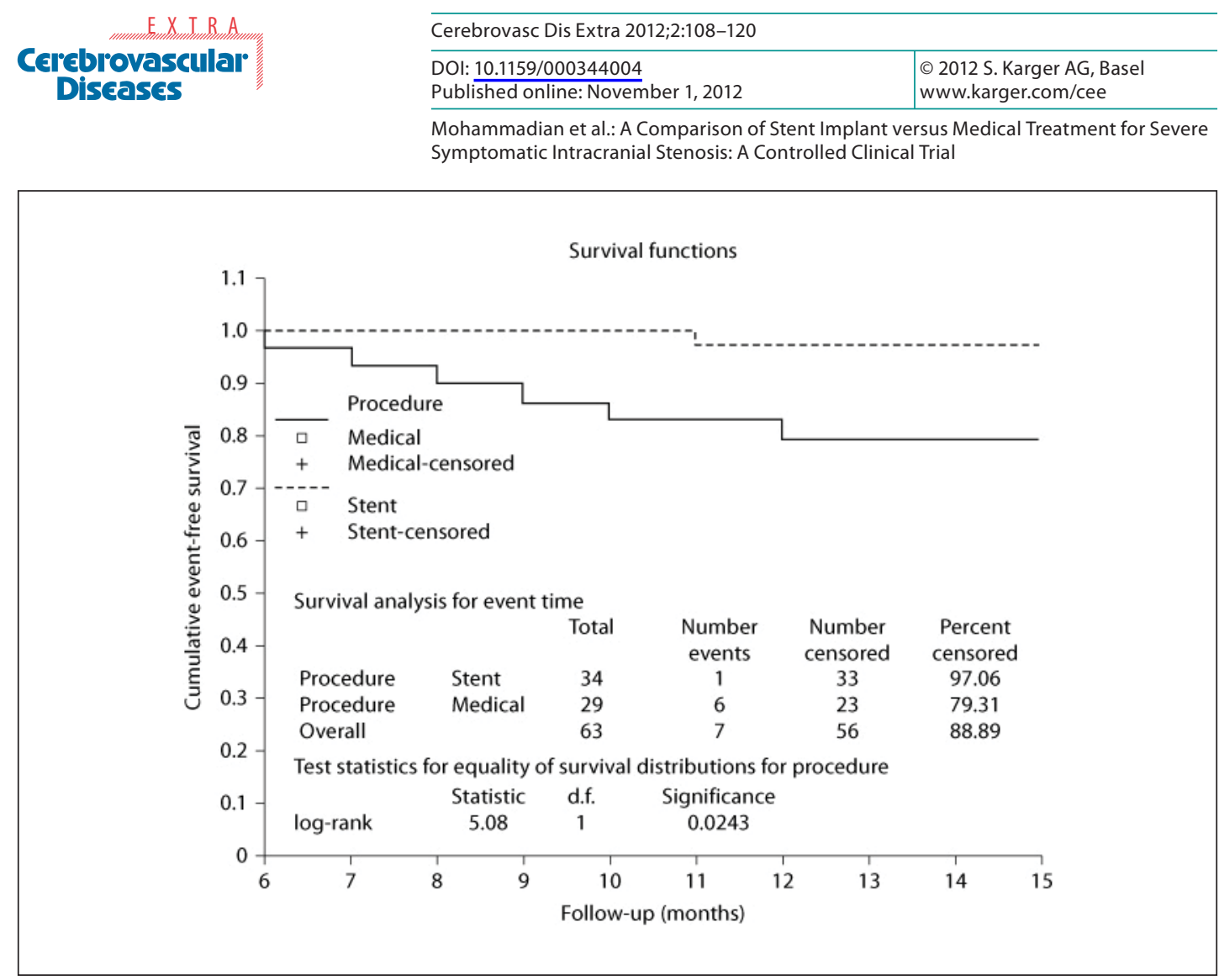

\begin{tabular}{|llllllll|}
\hline $\begin{array}{l}\text { Time } \\
\text { interval } \\
\text { months }\end{array}$ & & $\begin{array}{l}\text { Patients } \\
\text { entering this } \\
\text { interval, } \mathrm{n}\end{array}$ & $\begin{array}{l}\text { Patients with- } \\
\text { drawn during } \\
\text { interval, } \mathrm{n}\end{array}$ & $\begin{array}{l}\text { Patients } \\
\text { exposed } \\
\text { to risk, } \mathrm{n}\end{array}$ & $\begin{array}{l}\text { Secondary } \\
\text { AEs, } \mathrm{n}\end{array}$ & $\begin{array}{l}\text { Proportion } \\
\text { of event-free } \\
\text { survival }\end{array}$ & $\begin{array}{l}\text { Cumulative probability } \\
\text { of event-free survival at } \\
\text { end of interval, } \%\end{array}$ \\
\hline \multirow{2}{*}{$0-2$} & Medical: & 29 & 0 & 29 & 0 & 1.00 & 100 \\
& Stent: & 34 & 0 & 34 & 0 & 1.00 & 100 \\
\hline \multirow{2}{*}{-4} & Medical: & 29 & 0 & 29 & 0 & 1.00 & 100 \\
& Stent: & 34 & 0 & 34 & 0 & 1.00 & 100 \\
\hline \multirow{2}{*}{-6} & Medical: & 29 & 0 & 29 & 0 & 1.00 & 100 \\
& Stent: & 34 & 0 & 34 & 0 & 1.00 & 100 \\
\hline $6-8$ & Medical: & 29 & 0 & 29 & 2 & 0.931 & 93.1 \\
& Stent: & 34 & 0 & 34 & 0 & 1.00 & 100 \\
\hline $8-10$ & Medical: & 27 & 0 & 27 & 2 & 0.925 & 86.2 \\
& Stent: & 34 & 0 & 34 & 1 & 0.970 & 97.0 \\
\hline $10-12$ & Medical: & 25 & 0 & 25 & 1 & 0.960 & 82.7 \\
& Stent: & 33 & 0 & 33 & 0 & 0.970 & 97.0 \\
\hline $12-14$ & Medical: & 24 & 0 & 24 & 1 & 0.958 & 79.3 \\
& Stent: & 33 & 33 & 16 & 0 & 0.970 & 97.0 \\
\hline $14-16$ & Medical: & 23 & 23 & 11 & 0 & 1.00 & 79.3 \\
& Stent: & 33 & 33 & 16 & 0 & 0.970 & 97.0 \\
& & & & & & & \\
\hline
\end{tabular}

Fig. 2. Kaplan-Meier curve and table of cumulative secondary AE-free survival including mortality. Note that all AEs occurred within 6-12 months after enrolment of patients.

more acceptable than other reported results. The periprocedure ischemic stroke in our study occurred in a 63 -year-old female with a history of diabetes mellitus, hypertension, hyperlipidemia, and cigarette smoking. She had an ischemic stroke (mRS 3) during the first week after her procedure. The reported success rates of implantation of intracranial stents have been $>95 \%[23,24]$. Henkes et al. [25] investigated stent implantation and reported that the technical success rate was $97 \%$. The technical success rate for stent implantation was $97 \%$ in 
our study, which was in accordance with previously reported success rates [an unsuccessful stent implantation occurred in our patient who had residual stenosis (60\%) in the MCA]. The ischemic stroke and mortality rates after the procedure have ranged from 3.2 to $10 \%$ [26-28]. Suh et al. [29] reported on cases with intracranial stenosis that were $\geq 70 \%$ and were treated with various types of BMS. Their reported re-stenosis rate was $0 \%$ and the ischemic stroke and mortality rate was 10\% within 6 months of follow-up [29]. In the SSYLVIA trial, after applying BMSs, strokes occurred in $6.6 \%$ of the treated patients within 30 days and in $7.3 \%$ between 30 days and 1 year. Re-stenosis occurred in 35\% of the patients within 6 months after the procedure [30]. Gröschel et al. [20] performed a systematic review of outcomes after stenting for intracranial atherosclerosis and the re-stenosis rates were 34.6 and $16.1 \%$ for SES and BMS, respectively. Interestingly, our AE rates were lower than reported in these studies and there was no statistical significance in the re-stenosis rates between SESs (6.2\%) and BMSs (5.5\%). However, the BMS had a significant limitation because there was a technically difficult delivery to the convoluted intracranial vessels. The lower rates of re-stenosis and AEs in these cases could reflect the proper modification of risk factors and the attentiveness of our team. In the present investigation, all AEs in stent implant patients occurred within 6-12 months after the procedure, suggesting little profit in delayed stent implantation after 1 year.

Jarvis et al. [31] demonstrated that stent implant patients with 70-99\% stenosis had better stroke or mortality rates during the initial 30 days. They also had a better ipsilateral stroke rate beyond 30 days in comparison with the medically managed group [31], which was in accordance with our results. Dramatically, the stent implant group had a significantly lower unfavorable functional outcome rate than medically treated cases (fig. 1). This might arise from superior compliance in vascular risk factor control, ameliorated cerebrovascular autoregulation, scant in-stent re-stenosis, and a lower incidence of AEs. In contrast to the abovementioned results, Samaniego et al. [32] revealed a similar combined ischemic event rate and unfavorable functional outcome rate in medically managed and stent implant patients with intracranial stenosis. It was demonstrated that patients with poor control of risk factors, such as elevated low-density lipoprotein and blood pressure, had higher rates of recurrent vascular events [33-35].

A lower rate of ischemic stroke in stent implant cases versus medically managed cases (2.9 vs. $20.7 \%$ ) was obtained with the same optimal management of blood pressure and lipid profiles in both groups. Patients in the WASID trial who were treated with aspirin or warfarin and standard management of risk factors had a 10.7\% 30 -day rate of stroke or death and a 25\% 1-year rate for primary events [9]. In our medically managed patients, the 30-day rate of stroke or death and 1-year stroke rate were 0 and $13.8 \%$, respectively, which is lower than prior results. One possible explanation is that the combination of aspirin and clopidogrel played an important role in reducing the early risk of stroke. This conclusion is in accordance with the results of Wong et al. [36]. Because patients with 70-99\% stenosis are at the highest risk for complications with medical therapy rather than stent implantation, the benefit of stenting is likely to be greater in the severe stenosis group [37, 38]. Kasner et al. [37] demonstrated that patients with stenosis of $\geq 70 \%$ of a major intracranial artery, despite medical therapy and standard vascular risk factor modification, had a recurrent stroke rate of $23 \%$ at 1 year, particularly in the territory of the stenotic artery, which is in agreement with the above results.

\section{Study Limitations}

The comparison of stent implant and medically managed groups was done using univariate analysis. There are some limitations in our study. The number of patients was small (due to a limitation of eligible patients during the study period). Also, we excluded some pa- 


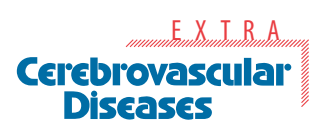

Cerebrovasc Dis Extra 2012;2:108-120

tients and therefore missed a number of patients who were eligible for stent placement or medical treatment. For this reason, we could not evaluate the predictive factors of morbidities.

\section{Conclusion}

The total event rate with severe intracranial stenosis in the stent implant group was lower than in the medically managed group during a mean period of 15.22 months of follow-up. The final functional outcome was better in the stent implant group, partially due to the lower occurrence of AEs. This study was not designed to confine the potential impact of risk factor management on long-term outcomes. Therefore, we suggest that endovascular angioplasty with stent implantation could be considered for patients with symptomatic severe intracranial stenosis of the ICA or MCA as a safer and more durable approach, despite optimal medical therapy. A randomized, multicenter, parallel trial may be required to compare the clinical efficacy of these two treatment modalities for symptomatic intracranial stenosis.

\section{References}

1 Donnan GA, Fisher M, Macleod M, Davis SM: Stroke. Lancet 2008;371:1612-1623.

- 2 Thom T, Haase N, Rosamond W, Howard VJ, Rumsfeld J, Manolio T, et al: Heart disease and stroke statistics - 2006 update. Circulation 2006;113:e85-e151.

-3 Gorelick PB, Wong KS, Bae HJ, Pandey DK: Large artery intracranial occlusive disease: a large worldwide burden but a relatively neglected frontier. Stroke 2008;39:2396-2399.

4 Wong LK: Global burden of intracranial atherosclerosis. Int J Stroke 2006;1:158-159.

-5 Lau AY, Wong EH, Wong A, Mok VC, Leung TW, Wong KS: Significance of good collateral compensation in symptomatic intracranial atherosclerosis. Cerebrovasc Dis 2012;33:517-524.

-6 Turan TN, Derdeyn CP, Fiorella D, Chimowitz MI: Treatment of atherosclerotic intracranial arterial stenosis. Stroke 2009;40:2257-2261.

-7 Chimowitz MI, Lynn MJ, Derdeyn CP, Turan TN, Fiorella D, Lane BF, et al: Stenting versus aggressive medical therapy for intracranial arterial stenosis. N Engl J Med 2011;365:993-1003.

-8 Abou-Chebl A, Steinmetz H: Critique of 'Stenting versus aggressive medical therapy for intracranial arterial stenosis'. Stroke 2012;43:616-620.

-9 Chimowitz MI, Lynn MJ, Howlett-Smith H, Stern BJ, Hertzberg VS, Frankel MR, et al: Comparison of warfarin and aspirin for symptomatic intracranial arterial stenosis. N Engl J Med 2005;352:13051316.

10 Mori T, Fukuoka M, Kazita K, Mori K: Follow-up study after intracranial percutaneous transluminal cerebral balloon angioplasty. Am J Neuroradiol 1998;19:1525-1533.

-11 Ziyal IM, Ozgen T, Sekhar LN, Ozcan OE, Cekirge S: Proposed classification of segments of the internal carotid artery: anatomical study with angiographical interpretation. Neurol Med Chir (Tokyo) 2005;45:184-191.

-12 Qureshi AI, Kirmani JF, Harris-Lane P, Divani AA, Ahmed S, Ebrihimi A, et al: Vertebral artery origin stent placement with distal protection: technical and clinical results. Am J Neuroradiol 2006; 27:1140-1145.

13 Samuels OB, Joseph GJ, Lynn MJ, Smith HA, Chimowitz MI: A standardized method for measuring intracranial arterial stenosis. AJNR Am J Neuroradiol 2000;21:643-646.

- 14 Higashida RT, Meyers PM, Connors JJ, Sacks D, Strother CM, Barr JD, et al: Intracranial angioplasty and stenting for cerebral atherosclerosis: a position statement of the American Society of Interventional and Therapeutic Neuroradiology, Society of Interventional Radiology, and the American Society of Neuroradiology. Am J Neuroradiol 2005;26:2323-2327. 


\begin{tabular}{|c|c|}
\hline Cerebrovasc Dis Extra 2012;2:108-120 & \\
\hline $\begin{array}{l}\text { DOI: } 10.1159 / 000344004 \\
\text { Published online: November 1, } 2012\end{array}$ & $\begin{array}{l}\text { (c) } 2012 \text { S. Karger AG, Basel } \\
\text { www.karger.com/cee }\end{array}$ \\
\hline
\end{tabular}

Mohammadian et al.: A Comparison of Stent Implant versus Medical Treatment for Severe Symptomatic Intracranial Stenosis: A Controlled Clinical Trial

-15 Qureshi AI, Feldmann E, Gomez CR, Johnston SC, Kasner SE, Quick DC, et al: Consensus conference on intracranial atherosclerotic disease: rationale, methodology, and results. J Neuroimaging 2009; 19:1S-10S.

-16 Higashida RT, Meyers PM, Connors JJ, Sacks D, Strother CM, Barr JD, et al: Intracranial angioplasty and stenting for cerebral atherosclerosis: a position statement of the American Society of Interventional and Therapeutic Neuroradiology, Society of Interventional Radiology, and the American Society of Neuroradiology. J Vasc Interv Radiol 2005;16:1281-1285.

-17 Marks MP: Is there a future for endovascular treatment of intracranial atherosclerotic disease after stenting and aggressive medical management for preventing recurrent stroke and intracranial stenosis (SAMMPRIS)? Stroke 2012;43:580-584.

-18 Qureshi AI, Feldmann E, Gomez CR, Johnston SC, Kasner SE, Quick DC, et al: Intracranial atherosclerotic disease: an update. Ann Neurol 2009;66:730-738.

-19 Choi JW, Kim JK, Choi BS, Lim HK, Kim SJ, Kim JS, et al: Angiographic pattern of symptomatic severe M1 stenosis: comparison with presenting symptoms, infarct patterns, perfusion status, and outcome after recanalization. Cerebrovasc Dis 2010;29:297-303.

-20 Gröschel K, Schnaudigel S, Pilgram SM, Wasser K, Kastrup A: A systematic review on outcome after stenting for intracranial atherosclerosis. Stroke 2009;40:e340-e347.

-21 Fiorella D, Chow MM, Anderson M, Woo H, Rasmussen PA, Masaryk TJ: A 7-year experience with balloon-mounted coronary stents for the treatment of symptomatic vertebrobasilar intracranial atheromatous disease. Neurosurgery 2007;61:236-242.

22 Cruz-Flores S, Diamond AL: Angioplasty for intracranial artery stenosis. Cochrane Database Syst Rev 2006;3:CD004133.

23 Jiang WJ, Srivastava T, Gao F, Du B, Dong KH, Xu XT: Perforator stroke after elective stenting of symptomatic intracranial stenosis. Neurology 2006;66:1868-1872.

24 Mazighi M, Yadav JS, Abou-Chebl A: Durability of endovascular therapy for symptomatic intracranial atherosclerosis. Stroke 2008;39:1766-1769.

-25 Henkes H, Miloslavski E, Lowens S, Reinartz J, Liebig T, Kuhne D: Treatment of intracranial atherosclerotic stenoses with balloon dilatation and self-expanding stent deployment (WingSpan). Neuroradiology 2005;47:222-228.

-26 Marks MP, Wojak JC, Al-Ali F, Jayaraman M, Marcellus ML, Connors JJ, et al: Angioplasty for symptomatic intracranial stenosis: clinical outcome. Stroke 2006;37:1016-1020.

-27 Bose A, Hartmann M, Henkes H, Liu HM, Teng MM, Szikora I, et al: A novel, self-expanding, nitinol stent in medically refractory intracranial atherosclerotic stenosis: the Wingspan study. Stroke 2007; 38:1531-1537.

28 Wolfe BF, Hussain SI, Lynch JR, Zaidat OO: Long term clinical and angiographic outcomes with the Wingspan stent for treatment of symptomatic 50-99\% intracranial atherosclerosis: single center experience in 51 cases. J Neurointerv Surg 2009;1:40-43.

-29 Suh DC, Kim JK, Choi JW, Choi BS, Pyun HW, Choi YJ, et al: Intracranial stenting of severe symptomatic intracranial stenosis: results of 100 consecutive patients. Am J Neuroradiol 2008;29:781-785.

-30 SSYLVIA Study Investigators: Stenting of symptomatic atherosclerotic lesions in the vertebral or intracranial arteries (SSYLVIA): study results. Stroke 2004;35:1388-1392.

31 Jarvis AL, Chimowitz M, Lynn MJ, Alexander MJ, et al; NIH Multicenter Wingspan Intracranial Stent Study Group: Outcome of patients with 50-99\% intracranial stenosis and TIA or stroke on antithrombotic therapy treated medically vs. stenting. Presented at: 60th Annual Meeting of the American Academy of Neurology, Chicago, IL, 2008.

-32 Samaniego EA, Hetzel S, Thirunarayanan S, Aagaard-Kienitz B, Turk AS, Levine R: Outcome of symptomatic intracranial atherosclerotic disease. Stroke 2009;40:2983-2987.

-33 PROGRESS Collaborative Group: Randomized trial of a perindopril-based blood-pressure-lowering regimen among 6,105 individuals with previous stroke or transient ischemic attack. Lancet 2001;358: 1033-1041.

-34 Chaturvedi S, Turan TN, Lynn MJ, Kasner SE, Romano J, Cotsonis G, et al; WASID Study Group: Risk factor status and vascular events in patients with symptomatic intracranial stenosis. Neurology 2007;69:2063-2068.

-35 Turan TN, Cotsonis G, Lynn MJ, Chaturvedi S, Chimowitz M; Warfarin-Aspirin Symptomatic Intracranial Disease (WASID) Trial Investigators: Relationship between blood pressure and stroke recurrence in patients with intracranial arterial stenosis. Circulation 2007;115:2969-2975. 
-36 Wong KS, Chen C, Fu J, Chang HM, Suwanwela NC, Huang YN, et al: Clopidogrel plus aspirin versus aspirin alone for reducing embolisation in patients with acute symptomatic cerebral or carotid artery stenosis (CLAIR study): a randomized, open-label, blinded-endpoint trial. Lancet Neurol 2010;9:489-497.

-37 Kasner S, Chimowitz M, Lynn M, Howlett-Smith H, Stern BJ, Hertzberg VS, et al: Predictors of ischemic stroke in the territory of a symptomatic intracranial arterial stenosis. Circulation 2006;113: 555-563.

- 38 Jiang WJ, Xu XT, Du B, Dong KH, Jin M, Wang QH, et al: Comparison of elective stenting of severe vs. moderate intracranial atherosclerotic stenosis. Neurology 2007;68:420-426. 